\title{
Analysis of Application Scenarios of Electronic Decision Theater in the Military Field
}

\author{
Xian Zhang ${ }^{1}$, Qi Xue ${ }^{2 *}$ \\ ${ }^{1}$ Center for Assessment and Demonstration, Academic of Military Sciences, Beijing, 100091, China \\ ${ }^{2}$ Center for Assessment and Demonstration, Academic of Military Sciences, Beijing, 100091, China
}

\begin{abstract}
The Decision Theater(DTs) is an immersive collaborative decision-making visualization solution, which plays a very important role in improving the scientific level of decision making. Based on the analysis of the technical origins, design concepts, application fields, and technical characteristics of the DTs, a general system architecture for the DTs for the military field is proposed, four typical application scenarios were discussed, including drafting and argumentation of major military decisions, simulation of military action plans, training of senior commanders' professional skills, and emergency response decision-making, which is of great value for improving the scientific nature of military decision-making.
\end{abstract}

\section{Introduction}

The "Recommendations on Formulating the Fourteenth Five-Year Plan for National Economic and Social Development and the Long-Term Goals for 2035" mentioned that it is necessary to improve the preevaluation and post-evaluation systems for major policies, unblock the channels for participating in policy formulation, and improve decision-making level of socialization, democratization, and rule of law. For the field of military full of confrontation and competition, whether it is the formation of various major military decision-making processes or the organization and implementation of major military combat exercises, it is necessary to handle scientific, democratic, and efficient decision-making issues. It is necessary to compare and analyse different decision-making schemes, and perform and simulate the main program methods. Based on the study of the DTs. we conduct a targeted analysis in combination with the main characteristics of the military field, which is of great value for improving the scientific nature of military decision-making.

\section{Current Research Status of Electronic Decision Theater}

\subsection{The origin of electronic decision theater}

The DTs develops custom software solutions to build scenarios, visualize tradeoffs and discover solutions across a wide range of social and organizational systems [1]. DTs can be viewed as meeting rooms characterized by specific input/display technologies and seating arrangement that allow a group of people to interact with each other and with the data in order to contextualize a decision-making situation, evaluate the impacts of decisions, and find a common solution.

The world's first electronic Decision Theater was built by the Public Decision Laboratory of Arizona State University in 2004[2]. which is suitable for specific decision-making problems, by making some assumptions around a hypothesis and predicting or simulating possible options, the decision makers can collectively solve the problem. The core component of the DTs is called "The Drum". The Drum can accommodate 25 people at the same time, with various tools for collecting input from participants, the three-dimensional animation demonstrations can be simultaneously used. Participants can watch the behaviour through three-dimensional glasses and evaluate various scenarios for future scenarios. Huazhong University of Science and Technology built the world's largest DTs in 2010 by absorbing the technology of DTs of Arizona State University(ASU)[3]. The DTs consist of an eight-channel 240-degree ring screen system, a professional graphics workstation cluster, a virtual scene resource configuration management system, computer cluster with supercomputing capabilities, was able to interactive simulation and remote decision-making information transmission and exchange, and has super integration capabilities with computing power, it can provide decision-makers with an interactive threedimensional intuitive decision-making environment. The DTs of Harbin Institute of Technology built at the same time includes design planning system, high-performance computer cluster, video image distribution processing system, audio distribution processing system, central integrated control system, meeting room/decision-making discussion system, etc, which could complete various kinds of decision analysis and process deduction analysis.

"Corresponding author's e-mail: scarebuff@163.com 


\subsection{Frontiers of Research on Electronic Decision Theater}

Since the world's first DTs was built at ASU in 2004, The Global Electronic Decision Theater Alliance was established. The alliance consists of Huazhong University of Science and Technology, Wuhan University [4], Arizona State University, Massachusetts Institute of Technology, and George City University, Renmin University of China, Harbin Institute of Technology and more than ten well-known universities at home and abroad [5]. A major joint R\&D team composed of different universities has been formed. The research fields cover socioeconomics, mathematics, computer software and hardware, and virtual simulation, many scientific decision-making system platforms was developed.

The ASU DTs has completed more than 70 cases, including the Phoenix Water Supply Project, Forest Planning, Urban Climate Change, Urban Development Planning, and the Scientific Design of School Curriculum, covering urban planning, intelligent transportation, Environmental resources, smart buildings, social economy and many other public management decisionmaking fields. The DTs of Huazhong University of Science and Technology has been used in government public decision-making, education services and supervision, urban planning, construction, supervision and traffic modelling. The CITIC Electronic DTs has formed a DTs for scientific and technological policy evaluation. It has been used in the evaluation of innovative cities, the perception of user needs of Chinese scientific and technological information resources, the evaluation of listed companies, and the management of emergency warnings.

\subsection{The Core technology of DTs}

The DTs integrates multiple disciplines and forms of expertise to leverage and manage the tension between differences, rather than compartmentalize them. The working process of DTs can be abstracted as a process from simulation, to three-dimensional visualization, and finally to group decision-making. The following six technologies have been comprehensively used:

Firstly, scenario-based gaming and simulations. Participants role play what-if scenarios by engaging with DTs' game and simulation-based learning techniques. An environment was created which allow participants to explore and understand the consequences and impacts of different levels of risk[6].

Secondly, Software. DTs uses an in-house, open source complex systems framework software platform.

The platform powers multi-screen model interaction to visually follow the effects and consequences of decision alternatives as they ripple throughout an integrated system. DTs constantly reduces cycle time and costs through increasingly growing capabilities and a mature technology shelf.

Thirdly, data analytics. DTs data analytics capabilities leverage staff expertise in geographical information systems and spatial optimization/modelling, social network analysis, statistical analysis, data mining, agent based modelling, machine learning, textual analysis, social media analysis, to customize solutions for a variety of innovative projects across a wide variety of topic areas.

Fourthly, user experience and user interaction. DTs models are designed to communicate the intricacies of a problem as well as the broader landscape of consequential action. DTs builds custom graphic dashboards for users to engage with databases, analytics and computations in realtime. Projects developed with DTs empower non-experts to engage in research, data exploration and computational modelling [6].

Fifthly, rapid data visualization. DTs can be utilized as a final product, as a way to explore data, or as a step in the iterative process for a larger project. Rapid data visualizations can take the form of interactive dashboards, exploratory data maps, timelines, or a combination of them and many others.

Sixthly, high-performance computing. The use of high-performance computing supports the integration of a wide range of computationally intensive models in various fields, including genetics, weather forecasting and climate research, enabling near-real-time decision-making.

\section{The Analytical Framework in the Military Field}

Compared with the public policy field of local governments, the military field has wider coverage, stronger antagonism, and greater risk in decision-making. Especially in the process of drafting and making major military decisions and organizing and implementing major exercises, it faces complex decision-making issues. It is necessary to reach a consensus on the content of the main decision-making matters in a short time, and make a scientific decision from a variety of different decisionmaking options. So we can design the following analysis framework.

\subsection{General Architecture of DTs}




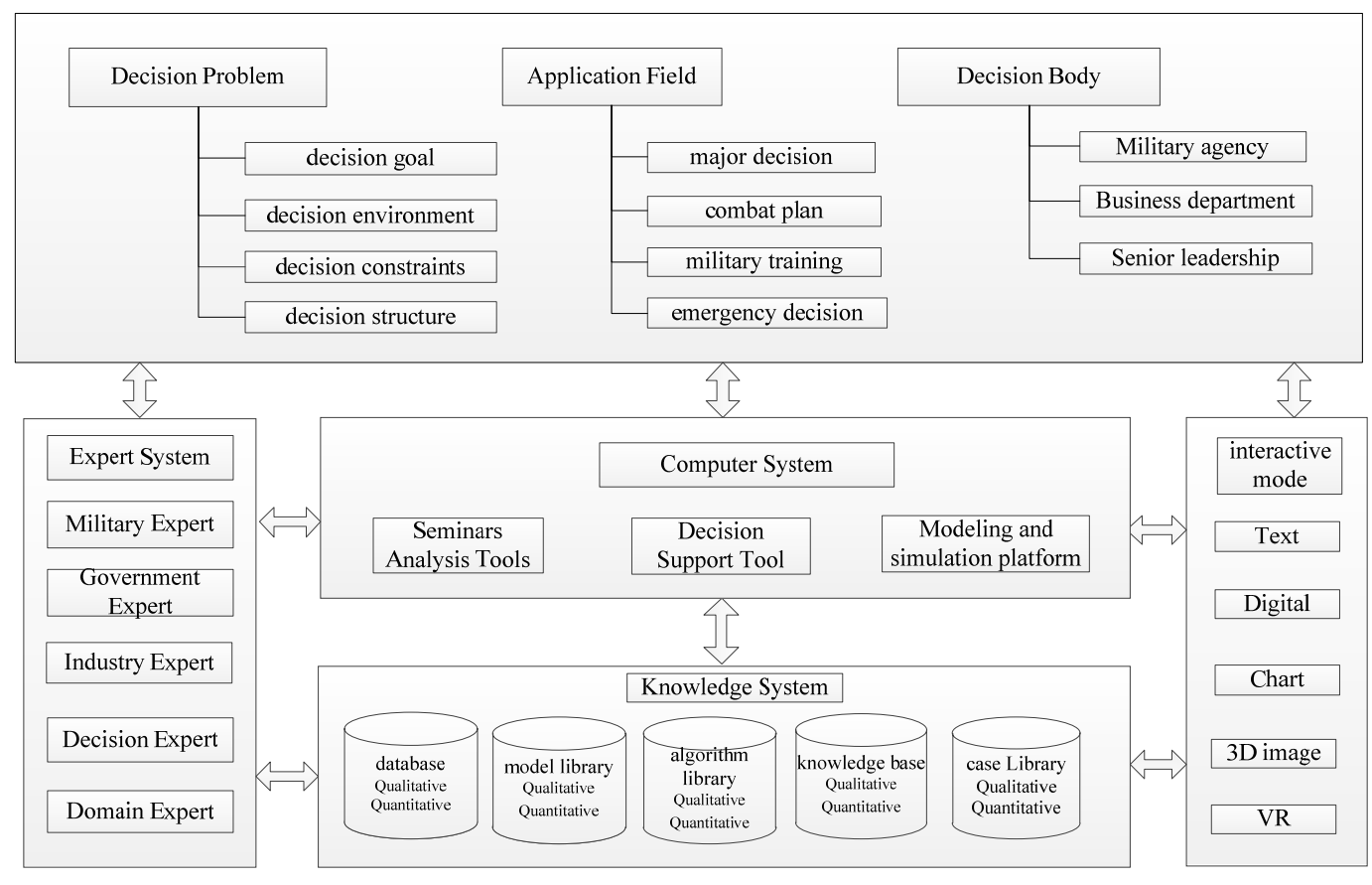

Figure 1. General Architecture of DTs

The DTs mainly includes three components: The Expert System, The Knowledge System, and The Computer System[7]. The Knowledge System refers to the sum of various intelligence information and fact data related to decision-making activities, including previous experience, domain knowledge, problem solving knowledge. The four types of knowledge are specifically embodied as databases, model libraries, algorithmic paths, knowledge bases, and case libraries that combine qualitative analysis and quantitative analysis. The Expert System, is aimed at the participants of the seminar, including various experts directly related to decision-making, including military commissioners, department leaders, industry experts, decision-making experts, and domain experts. The Computer Service System refers to the construction of computer supporting conditions in the integrated seminar environment. Specifically, it refers to the use of computer data storage capabilities, information processing capabilities, and knowledge presentation capabilities to collect, analyse, and process the opinions of seminar participants, so that many participants Opinions can be integrated and fed back into the system, and the ability to obtain new results through the system.

\subsection{Application Scenarios of DTs}

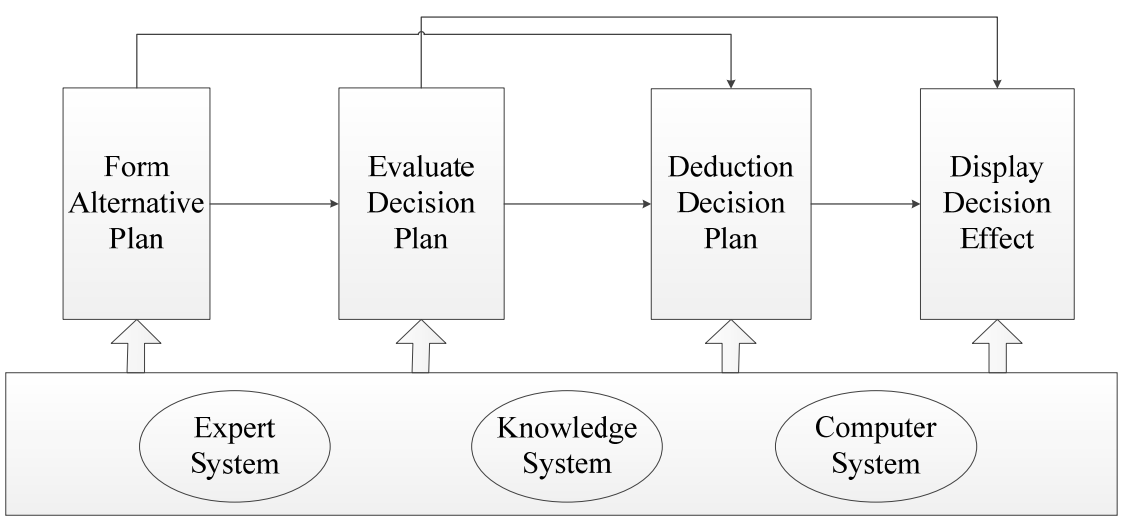

Figure 2. Application Scenarios of DTs

The DTs is consisted of The Expert System, The Knowledge System and The Computer System. According to the decision-making process, there are mainly the following four application scenarios[8-10]: The first is the analysis of alternative decision-making schemes, based on the expert experience system, the idea of forming alternative schemes through the analysis of existing cases, and the use of scientific modelling analysis tools to form alternative schemes on this basis. The second is the evaluation of alternatives, through the design of decision-making models and the use of technical indicators to analyse the pros and cons of multiple alternatives. The third is to perform deductions on the decision-making plans, through model adjustment, parameter adjustment and human-computer interaction deduces the implementation of alternative plans. Fourth, the effect of decision-making plans is displayed. Virtual simulation technology is used to comprehensively display 
the effects of a single set of decision-making plans and multiple sets of decision-making plans.

\subsection{The basic analysis process of DTs}

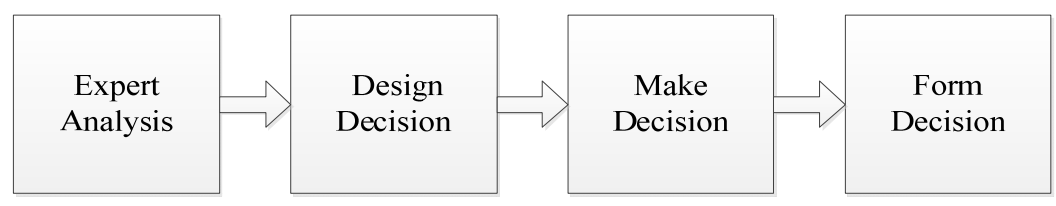

Figure 3. The basic analysis process of DTs
For general decision-making problems, decisions can be made by the four stages.

In the qualitative analysis stage, the relevant decisionmaking issues are first raised, experts are grouped to discuss the issues, and the issues are structured and an evaluation index system is created.

In the plan design stage, after creating a basic scenario based on the data, and construct an index system to solve the scenario, the initial data was entered to generate multiple sets of plans.

In the decision-making stage, firstly organize relevant personnel to hold a decision-making meeting, pour into multiple sets of plans for comparison and analysis, and then modify the impact factor based on expert opinions, generate the latest calculation results according to the calculation model, use the DTs to display the decision results, use the voting system to make the final decision.

In the plan formation stage, the generated decision plan is stored in the knowledge base, the storage model is imported into the model library, the final results and query opinions are released through the information release system.

\section{The possible applications in the military field}

The unique immersive visual experience of the DTs, the front-end and back-end cooperative operation mode, the completion of diversified decision-making tasks, the advantages of effectively stimulating the image thinking, determines its great application scenarios in the military field.

\subsection{Drafting for major military decisions}

In the process of drafting and demonstrating major military decisions, the main decision-making texts are detailed and decomposed to form a list of relevant decision-making matters. The selection part was focused on and conducted according to the responsible parties involved in the specific decision-making clauses, the human-in-the-loop mode was used to judge the pros and cons of the decision-making plan. Through the immersive interactive experience of experts on the main decisionmaking issues, the decision-making risks are reduced and the decision-making quality was improved.

\subsection{Simulation of military action plan}

In the deduction of major military action plans, especially for the main links and content of the combat plan, the DTs can transform the decision model into a decision script, adopt modelling and simulation technology to model and analyse the combat space, combat forces, and combat actions, adopt the virtual reality technology to construct a virtual battlefield environment, integrate the commanders into the virtual reality environment, and complete the deduction simulation of the combat action plan based on the simulation deduction engine.

\subsection{Professional skills training for senior commanders}

During the professional training of senior commanders, the ability and quality required by senior commanders are transformed into specific index content, and a list of optional operating procedures can design and deduct in the DTs. The DTs can not only complete the assessment of the comprehensive ability and quality of the senior commanders, but also complete the assessment of the individual professional ability of the senior commanders, and improve the professional ability level of the senior commanders through interactive assessment.

\subsection{Emergency response decision-making process}

In the process of emergency response decision-making, different emergency decision-making and disposal are transformed into different decision-making scripts. According to the main body involved in the decisionmaking relationship, occurrence probability and time sequence connection of various decision-making activities, by designing a multi-attribute decision model, According to user interaction, the pros and cons of various decision-making schemes are sorted.

\section{Conclusion}

Based on the analysis of the origin, design idea, application field and technical characteristics of DTs, we propose a general system architecture of DTs suitable for the military field, and discusses four different application scenarios. It is of great reference value to enhance the indepth integration of decision-making in the military field. 


\section{References}

1. Shutters, S.T.; Kandala, S.S.; Wei, F.; Kinzig, A.P (2021). Resilience of Urban Economic Structures Following the Great Recession. Sustainability, 13, 2374.

2. Miller, J., Salla, R., Amresh, A., Smith, H., Kandala, S., Hinrichs, M., Gorantla, R., Sokteva, E., Wei, F., Hirsch, K., \& others. (2019). The Decision Theater: Collaborative Research Methodology.

3. Mao Zijun, Xu Xiaolin, Xu Xiaodong (2018). Electronic Decision Theater for Scientific and Democratic Decision Navigation, Chinese Administration, 2011, Issue 9,120-122.

4. White, D. D., Larson, K. L., \& Wutich, A. (2018). Boundary Organizations and Objects Supporting Stakeholders for Decision Making on Sustainable Water Management in Phoenix, Arizona USA. In Transformations of Social-Ecological Systems (pp. 333-352). Springer, Singapore.

5. White, D., Jones, J., Maciejewski, R., Aggarwal, R., \& Mascaro, G. (2017). Stakeholder analysis for the food-energy-water nexus in phoenix, Arizona:
Implications for nexus governance. Sustainability, 9(12), 2204.

6. https://dt.asu.edu/technology.

7. Wang Xinglin (2008). The Decision-making mechanism of Decision Theater under the sciences of public policy. Harbin Institute of Technology.

8. Boukherroub, T., D’AMOURS, S., \& Rönnqvist, M. (2016). Toward decision theater design for community forest management \& planning: The case of Québec. In 11th International Conference on Modeling, Optimization and Simulation, Montréal, Québec, Canada.

9. Gober, P. (2018). Social Learning for Water Sector Resilience. In Building Resilience for Uncertain Water Futures (pp. 163-190).

10. Amresh, A., Salla, R., Sinha, M., \& Birr, R. (2016). Design, implementation and evaluation of a gamebased intervention targeting latino children for improving obesity outcomes. In 2016 IEEE International Conference on Serious Games and Applications for health (SeGAH) (pp. 1-8). IEEE. 